\title{
Taxones superiores de hormigas como sustitutos de la riqueza de especies, en una cronosecuencia de bosques secundarios, bosque primario y sistemas agroforestales en la Amazonía Oriental, Brasil
}

\author{
Jhonatan Andrés Muñoz Gutiérrez ${ }^{1}$, Guillaume Xavier Rousseau ${ }^{1 *}$, Joudellys Andrade-Silva ${ }^{2}$ \& \\ Jacques Hubert Charles Delabie ${ }^{3,4}$ \\ 1. Programa de Pós-Graduação em Agroecologia, Universidade Estadual do Maranhão, São Luís, MA, Brasil. CEP \\ 65055-970; energiaselvatica@gmail.com, guilirous@yahoo.ca \\ 2. Programa de Pós-Graduação em Biodiversidade e Conservação, Universidade Federal do Maranhão, São Luis, MA, \\ Brasil. CEP 65085-580; jascb09.2@gmail.com \\ 3. Comissão Executiva do Plano da Lavoura Cacaueira, Centro de Pesquisas do Cacau, Laboratório de Mirmecologia - \\ CEPEC/CEPLAC. Itabuna, BA, Brasil. CEP 45650-000; jacques.delabie@gmail.com \\ 4. Universidade Estadual de Santa Cruz. Ilhéus, BA, Brasil. \\ * Correspondence
}

Recibido 28-III-2016. C Corregido 08-VIII-2016. Aceptado 09-IX-2016.

\begin{abstract}
Ants' higher taxa as surrogates of species richness in a chronosequence of fallows, old-grown forests and agroforestry systems in the Eastern Amazon, Brazil. Deforestation in Amazon forests is one of the main causes for biodiversity loss worldwide. Ants are key into the ecosystem because act like engineers; hence, the loss of ants' biodiversity may be a guide to measure the loss of essential functions into the ecosystems. The aim of this study was to evaluate soil ant's richness and to estimate whether higher taxa levels (Subfamily and Genus) can be used as surrogates of species richness in different vegetation types (fallows, old-growth forests and agroforestry systems) in Eastern Amazon. The samples were taken in 65 areas in the Maranhão and Pará States in the period 2011-2014. The sampling scheme followed the procedure of Tropical Soil Biology and Fertility (TSBF). Initially, the vegetation types were characterized according to their age and estimated species richness. Linear and exponential functions were applied to evaluate if higher taxa can be used as surrogates and correlated with the Pearson coefficient. In total, 180 species distributed in 60 genera were identified. The results showed that ant species richness was higher in intermediate fallows (88) and old secondary forest (76), and was lower in agroforestry systems (38) and mature riparian forest (35). The genus level was the best surrogate to estimate the ant's species richness across the different vegetation types, and explained $72-97 \%(\mathrm{P}<0.001)$ of the total species variability. The results confirmed that the genus level is an excellent surrogate to estimate the ant's species richness in the region and that both fallows and agroforestry systems may contribute in the conservation of Eastern Amazon ant community. Rev. Biol. Trop. 65 (1): 279-291. Epub 2017 March 01.
\end{abstract}

Key words: surrogates, conservation, rapid richness assessment, Amazon, taxonomy.

La deforestación en la Amazonía brasileña, se considera como una de las principales causas de pérdida y reducción de biodiversidad (Laurance \& Vasconcelos, 2009; Fearnside, 2010). Se estima que el $20 \%$ del Bioma se ha perdido en Brasil (INPE, 2015), y la región conocida como Centro de Endemismo BelémCEB, donde se ubican los Estados Pará y Maranhão, es la más degradada. En el CEB, los remanentes de bosque primario ocupan apenas el $24 \%$ y existe un $18 \%$ del territorio en proceso de sucesión (Almeida \& Vieira, 2010). La ganadería extensiva y el monocultivo de soya, son los principales usos del suelo en esa región y las principales causas de la deforestación (Fearnside, 2010). Así, los bosques secundarios en sucesión, es decir, en proceso ecológico de recuperación de la cobertura boscosa original 
(Yepes, del Valle, Jaramillo, \& Orrego, 2010), y los sistemas agroforestales, se tornan de interés para la conservación de la biodiversidad (Rousseau, Rogério, Celentano, \& Carvalho, 2014) y el desarrollo sostenible.

La Amazonía alberga una diversidad elevada de organismos, aunque para muchos de ellos, son poco conocidos los aspectos fundamentales para la conservación biológica, como su distribución y riqueza (Grelle, 2002; Fearnside, 2006), lo que dificulta la conservación y manejo de este Bioma. Ese conocimiento es prácticamente imposible de ser alcanzado en su totalidad, porque depende de investigaciones onerosas, tiempo y recursos humanos (Vieira, Oliveira, Brewster, \& Gayubo, 2012; Neeson, Van Rijn, \& Mandelik, 2013). En este contexto, con la necesidad urgente de estimar la riqueza y distribución de especies de manera más simple y rápida (Cardoso, Silva, de Oliveira, \& Serrano, 2004; Rosser \& Eggleton, 2012), la herramienta de taxón superior (Gaston \& Williams, 1993) ha sido implementada con éxito en diferentes grupos de organismos (Sebastia \& Grelle, 2009; Kallimanis et al., 2012; Vieira et al., 2012). Sin embargo, la información es escasa respecto a invertebrados del suelo, por ser considerados taxones con alta diversidad, especialmente en la región tropical (Heino \& Soininen, 2007; Neeson et al., 2013).

Dentro de los invertebrados, las hormigas son consideradas como uno de los grupos sociales más representativos (Folgarait, 1998). Son de suma importancia dentro de la fauna edáfica, y en general de los ecosistemas, por su capacidad de interacción con otros organismos (Hölldobler \& Wilson, 1990). Igualmente, son consideradas ingenieras del suelo (Lavelle et al., 2006), por sus diversas funciones que inciden directa e indirectamente en la estructura y funcionamiento de los ecosistemas (Vasconcelos, 1999; Vasconcelos \& Laurance, 2005). Adicionalmente, debido a su resilencia ante perturbaciones antropogénicas, han sido utilizadas como grupo bioindicador de alteraciones ambientales (Pais \& Varanda, 2010; Ribas et al., 2012; Guzmán-Mendoza, Castaño-Meneses, \& Nuñez-Palenius, 2016).
A pesar de la vasta información sobre el estudio de las hormigas en diferentes campos del conocimiento, persiste un vacío de información básica, como la riqueza y la distribución de especies, en muchas regiones del neotrópico (Lattke, 2003), incluyendo los estados de Maranhão y Pará en el CEB. El presente estudio, tuvo como objetivo evaluar la riqueza de especies de hormigas del suelo en diferentes estados de sucesión del bosque y sistemas agroforestales en la Amazonía Oriental, y estimar si los taxones superiores (Subfamilia y Género) son buenos sustitutos de la riqueza de especies.

\section{MATERIALES Y MÉTODOS}

Área de estudio: El estudio se desarrolló en la Amazonía Oriental en ocho municipios, siete ubicados en el estado de Maranhão (Centro Novo do Maranhão, Bom Jardim, São Luís, São José de Ribamar, Rosário, Axixá y Alcântara) y uno en Pará (Tomé-Açú) (Apéndice). Los municipios de Alcântara, Centro Novo do Maranhão, Tomé-Açú y Bom Jardim hacen parte de la región denominada Centro de Endemismo Belén (CEB) Amazonía Oriental. El tipo de suelo predominante es el oxisol (Quesada et al., 2011), el cual presenta un perfil profundo de baja fertilidad y baja saturación de bases (IBGE, 2011). El clima regional es clasificado como tropical lluvioso Am en Centro Novo do Maranhão, Tomé-Açú y Bom Jardimm, y As en São Luís, São José de Ribamar, Alcântara, Rosário y Axixá. La elevación en la región comprende un rango entre 4 y $294 \mathrm{~m}$. La temperatura promedio en la región es de $27^{\circ} \mathrm{C}$ con precipitación promedio 2100 en Maranhão y 2300 mm/año en Tomé-Açú (Alvares, Stape, Sentelhas, Moraes-Gonçalves, \& Sparovek, 2013). El Tipo de vegetación presente corresponde a bosque ombrófilo denso en la región de Centro Novo do Maranhão, Bom Jardim y Tomé-Açú, ombrófilo abierto en São Luis, Axixá y Rosário, bosque ribereño ombrófilo abierto en Alcântara y bosque de Restinga en São José de Ribamar (IBGE, 2011). 
Diseño experimental y método de muestreo: Los muestreos fueron realizados entre 2011 y 2014, en 65 parcelas en diferentes estados de sucesión vegetal clasificadas de acuerdo con su edad (Apéndice): bosque primario (9 parcelas), bosque secundario avanzado 19-60 años (9), bosque secundario intermedio 11-16 años (9), bosque secundario joven 3-9 años (7), sistema agroforestal 9-36 (5), bosque ribereño primario (6), bosque secundario ribereño avanzado (6), bosque secundario ribereño joven (6), área abierta de uso agrícola (pastos misturados con yuca) (6) y bosque primario de Restinga (2). Las edades de las parcelas fueron proporcionadas por los dueños o usuarios de las mismas. Las parcelas consistieron en un círculo de $50 \mathrm{~m}$ de diámetro $(1953 \mathrm{~m})$, el cual se dividió en cuatro cuadrantes, donde, fueron recolectados cinco monolitos $25 \times 25 \times 10 \mathrm{~cm}$ de suelo utilizando el método Tropical Soil Biology and Fertility/ TSBF (Anderson \& Ingram, 1993) modificado, en forma de cruz a cada $20 \mathrm{~m}$, partiendo de un punto central (Rousseau et al., 2014). En Alcântara las parcelas fueron rectangulares $\left(1000 \mathrm{~m}^{2}\right)$ y paralelas al río. Estas parcelas fueron muestreadas dos veces en meses diferentes, donde en cada muestreo, cinco monolitos de suelo de $25 \times 25 \times 10 \mathrm{~cm}$, distribuidos en zig-zag a lo largo de la línea media de la parcela, fueron recolectados. La diferencia del área utilizada en Alcântara correspondió a la necesidad de integración a un protocolo de un proyecto con objetivos diferentes y a la adaptación del tamaño de los mosaicos presentes en la zona. Para más detalles ver Zelarayán et al. (2015). La hojarasca y el suelo de cada monolito fue procesada manual e independientemente, y toda la mirmecofauna recolectada fue conservada en alcohol al $70 \%$, directamente.

Identificación del material: La mirmecofauna recolectada, fue identificada siempre que fue posible, hasta especie o morfoespecie. La identificación de género se hizo con base en Palacio y Fernández (2003), y las especies fueron identificadas en el Laboratorio de Mirmecologia, del Centro de Investigación en Cacao CEPEC/CEPLAC, Itabuna/Bahía- Brasil. Los especímenes se depositaron en la colección de referencia (CPDC) del mismo laboratorio bajo el número de registro 5759 , así como en la sub-colección de invertebrados del suelo de Maranhão (en proceso de homologación), Universidad Estadual de Maranhão.

Todas las parcelas fueron agrupadas independientemente de su localización de acuerdo a su estado de sucesión vegetal. La riqueza observada de especies, se obtuvo a partir del número de especies o morfo-especies por estado de sucesión vegetal del bosque y sistemas agroforestales, y como estimador no paramétrico, fue utilizado Chao 2 con 100 randomizaciones sin remplazo usando datos de incidencia.

Por tratarse de un diseño con diferente esfuerzo de muestreo, fueron utilizados el método de rarefacción de Coleman (Coleman, 1981) interpolado a 25 monolitos (cinco parcelas) y curvas de rarefacción extrapoladas a 60 monolitos (Colwell et al., 2012). Los análisis se realizaron por medio del software EstimateS 9.1.0 (Colwell, 2013).

La estimación de la riqueza de especies por medio de los taxones sustitutos (subfamilia y género) para cada estado de sucesión vegetal y sistema agroforestal, fue realizada a través de funciones exponenciales para subfamilia y lineales para género. Se realizó prueba de normalidad de Shapiro-Wilk para los residuos y posteriormente se utilizó correlación de Pearson. Para definir si un sustituto era considerado como razonable (> $60 \%$ ), bueno $(>70 \%)$ o excelente estimador (>80\%), se utilizaron los valores propuestos por Leal, Bieber, Tabarelli y Andersen (2010). La vegetación de restinga sólo se utilizó en parte para el análisis total de las áreas, debido al bajo esfuerzo de muestreo. Las regresiones se realizaron usando el programa STATISTICA 7.0 (StatSoft, versión 2002).

\section{RESULTADOS}

Se identificó 180 especies en 60 géneros y nueve subfamilias. Las subfamilias más comunes fueron Myrmicinae $47.7 \%$, Ponerinae $22.6 \%$, Formicinae $15.3 \%$, que abarcaron $85.6 \%$ del total de las especies, mientras 
que los géneros con mayor número de especies fueron Pheidole $15.1 \%$, Hypoponera $14.1 \%$ y Solenopsis $10.3 \%$. La mayor riqueza total de especies, se registró en el bosque secundario intermedio (88 spp.) y avanzado (76), mientras los valores menores fueron encontrados en el bosque ribereño (35) y sistema agroforestal (38) (Cuadro 1, Fig. 3). El estimador Chao2 explicó entre el 47-83\% de la riqueza total.

Las curvas de rarefacción de especies extrapoladas para 60 (12 parcelas) monolitos no presentaron saturación o estabilidad (Fig. 1). Las regresiones indican una fuerte relación linear entre el género y la riqueza de especies $\left(\mathrm{r}^{2}=74-97 \%, \mathrm{P}<0.05\right)$ para todos los diferentes estados de sucesión del bosque y sistema agroforestal, así como considerando el total de las áreas $\left(\mathrm{r}^{2}=0.92 ; \mathrm{P}<0.001\right)$ (Fig. 2). Las regresiones indican que el género puede ser considerado un sustituto de especies excelente ( $>80 \%$ ) en BP, BSA, BSI, BRP, BSRA y AA, y bueno $(>70 \%)$ en BSJ, BSRJ y SAF. Por otro lado, aunque la subfamilia presentó una relación exponencial significativa con la riqueza total de hormigas $(\mathrm{P}<0.05)$, esa relación es considerada inadecuada $\left(\mathrm{r}^{2}=29 \%\right)$ para ser usada como sustituto. Además, cuando fue evaluada para cada sistema, la relación entre subfamilia y especie no fue significativa ( $\mathrm{P}<$ 0.05) para SAF, BSA, BSJ y BSI. Los sistemas que presentaron una relación significativa $(\mathrm{P}<$ $0.05)$ y de uso como sustituto razonable fueron AA (62\%), BSRJ (63\%).

\section{DISCUSIÓN}

La riqueza de especies de hormigas encontrada en este estudio es elevada, considerando

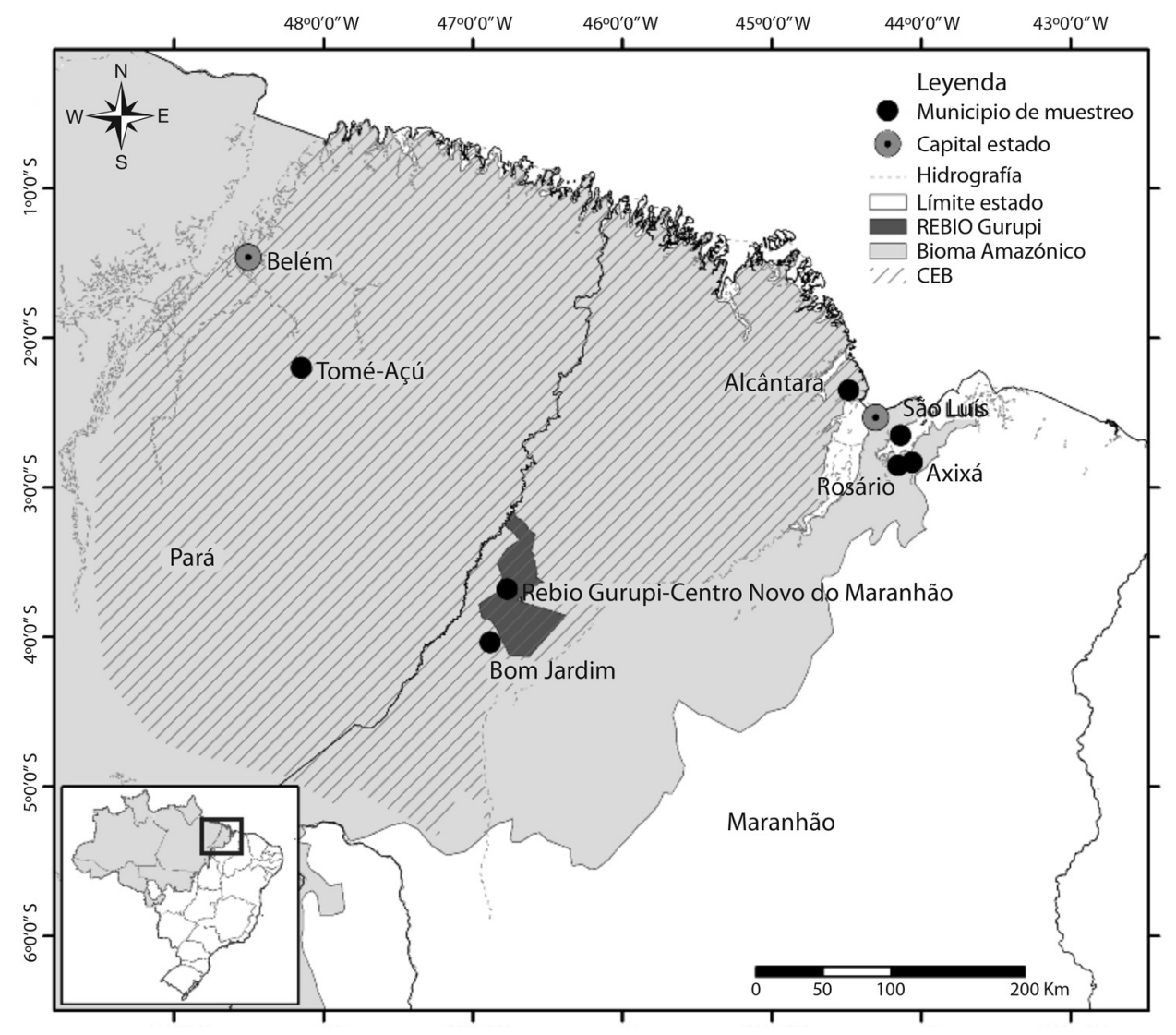

Fig. 1. Distribución geográfica de las áreas de estudio.

Fig. 1. Geographic distribution in the study areas. 
CUADRO 1

Riqueza de especies de hormigas en una cronosecuencia del bosque, bosque primario y sistema agroforestal en la Amazonía Oriental, Brasil

TABLE 1

Ant's species richness in a chronosequence of fallows, old-growth forests and agroforestry systems in the Eastern Amazon, Brazil

\begin{tabular}{|c|c|c|c|c|c|c|}
\hline Sucesión vegetal & $\begin{array}{c}\text { Observada } \\
\text { (S) }\end{array}$ & $\begin{array}{c}\text { Media } \\
\text { (S) }\end{array}$ & $\begin{array}{c}\text { Chao2 } \\
\text { (S) }\end{array}$ & $\begin{array}{c}\text { Explicado } \\
\%\end{array}$ & $\begin{array}{l}\text { Rarificada } \\
\text { (S) }\end{array}$ & $\begin{array}{c}\mathrm{N}^{\circ} . \\
\text { parcelas }\end{array}$ \\
\hline Bosque primario & 70 & $18.77 \pm 5.97$ & $84.37 \pm 7.86$ & 83 & $54.93 \pm 3.12$ & 9 \\
\hline Bosque secundario avanzado & 76 & $16.33 \pm 6.16$ & $109.37 \pm 15.7$ & 69.5 & $57.44 \pm 3.4$ & 9 \\
\hline Bosque secundario intermedio & 88 & $18.55 \pm 8.49$ & $149.07 \pm 26.34$ & 59 & $65.22 \pm 3.72$ & 9 \\
\hline Bosque secundario joven & 51 & $13 \pm 5.07$ & $69.08 \pm 10.13$ & 73.8 & $43.53 \pm 2.36$ & 7 \\
\hline Sistema agroforestal & 38 & $11.4 \pm 3.36$ & $80.34 \pm 27.24$ & 47.3 & 38 & 5 \\
\hline Bosque ribereño primario & 35 & $5.83 \pm 2.98$ & $41.92 \pm 5.07$ & 83.5 & $22.32 \pm 2.59$ & 12 \\
\hline Bosque secundario ribereño avanzado & 49 & $10.1 \pm 3.42$ & $83 \pm 20.63$ & 59 & $31.7 \pm 2.34$ & 12 \\
\hline Bosque secundario ribereño joven & 48 & $10.5 \pm 3.87$ & $61.77 \pm 9.75$ & 77.7 & $34.49 \pm 2.73$ & 12 \\
\hline Área abierta & 58 & $12.75 \pm 4.09$ & $78.01 \pm 10.97$ & 74.3 & $38.15 \pm 3.2$ & 12 \\
\hline
\end{tabular}

Riqueza (S): Observada, Media, Estimada (Chao 2), Rarificad, \pm Desviación estándar. Richness (S): Observed, Mean, estimated (Chao2), rarefied, \pm Standard Deviation.

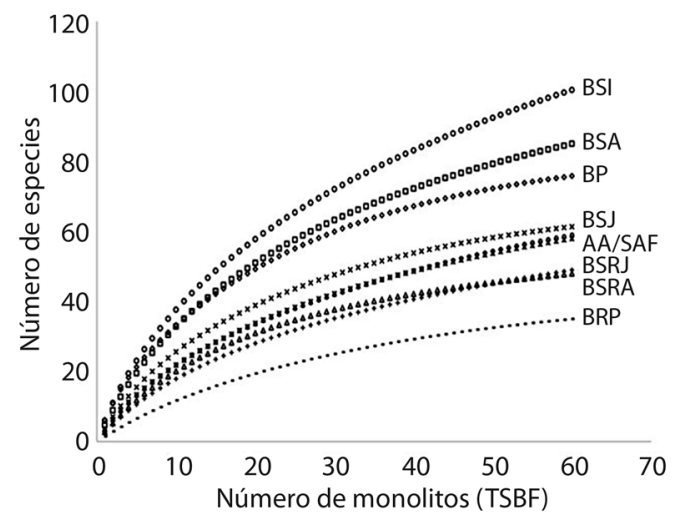

Fig. 2. Curvas de rarefacción de especies de hormigas extrapolada en: Bosque primario (BP) Bosque secundario avanzado (BSA), Bosque secundario intermedio (BSI), Bosque secundario joven (BSJ), Área abierta (AA), Sistema agroforestal (SAF), Bosque ribereño primario (BRP), Bosque secundario ribereño avanzado (BSRA) y Bosque secundario ribereño joven (BSRJ), en la Amazonía Oriental, Brasil.

Fig. 2. Species accumulation curve of ants extrapolated in: Mature forest (BP), Old secondary forest (BSA), Intermediated Secondary Forest (BSI), Young secondary forest (BSJ), Deforested area (AA), Agroforestry system (SAF), Riparian mature forest (BRP), Old secondary riparian (BSRA) Young secondary riparian forest (BSRJ), in the Eastern Amazon. que registró el $12.3 \%$ de las especies registradas para el Brasil (Antwiki, 2015), aún cuando se reconoce que el método de muestreo utilizado es diseñado para la macrofauna en general de suelo y no específicamente para las comunidades de hormigas (Sanabria, Lavelle, $\&$ Fonte, 2014). De igual manera, los resultados en este estudio son consistentes con otros trabajos que utilizaron diferentes métodos de muestreo y registraron la subfamilia Myrmicinae (Chanatásig-Vaca et al., 2011; Miranda, Oliveira, Baccaro, Morato, \& Delabie, 2012; Vergara-Navarro \& Serna, 2013), y los géneros Pheidole, Solenopsis (Santos et al., 2006; Wilkie, Mertl, \& Traniello, 2010; Freitas, Delabie, \& Lacau, 2014) e Hypoponera (Santos et al., 2006; Chanatásig-Vaca et al., 2011; Queiroz, Ribas, \& França, 2013) como grupos dominantes y diversos del suelo, en los ecosistemas neotropicales. Estos resultados se enmarcan dentro del contexto que estas subfamilias y géneros, dentro de los grupos de Formicidae, son considerados como megadiversos y dominantes (Antwiki, 2015). Esta dominancia según Fowler, Forti, Brandão, Delabie y Vasconcelos 

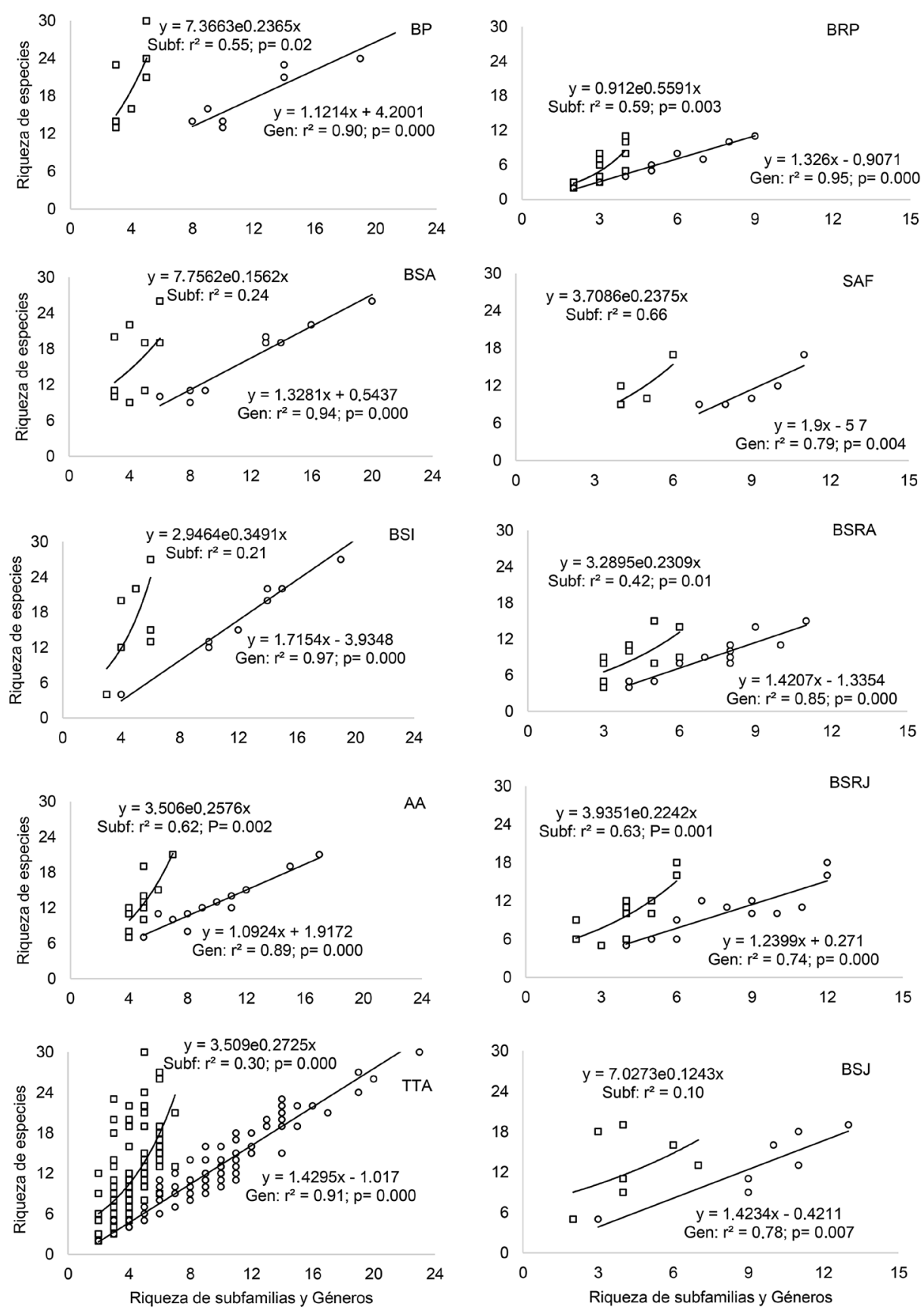

Fig. 3. Relación entre la riqueza de especies y la riqueza de géneros (Gen) y subfamilias (Subf) en hormigas en: Bosque primario (BP) Bosque secundario avanzado (BSA), Bosque secundario joven (BSJ), Bosque secundario intermedio (BSI), Área abierta (AA), Sistema agroforestal (SAF), Bosque ribereño primario (BRP), Bosque secundario ribereño avanzado (BSRA), Bosque secundario ribereño joven (BSRJ), y para el total de áreas (TTA) en la Amazonía Oriental, Brasil.

Fig. 3. Relationship between species richness and genus (Gen) and subfamily (Subf) richness of the ants in: Mature forest (BP), Old Secondary Forest (BSA), Young secondary forest (BSJ), Intermediated secondary forest (BSI), Deforested area (AA), Agroforestry system (SAF), Riparian mature forest (BRP), Old secondary riparian (BSRA), Young secondary riparian forest (BSRJ) and in all sampling sites (TTA) in the Eastern Amazon. 
(1991) y Fernández (2003) puede estar asociada a su plasticidad en hábitos alimenticios, tamaño y capacidad de dispersión de las especies.

Los bosques ribereños presentaron de manera general las menores riquezas. Esta situación se puede asociar con los periodos de anegamiento a los cuales están sometidas durante el periodo de lluvias. El anegamiento, genera el desplazamiento de la mirmecofauna hacia zonas más altas y secas, y ya fue observado en la región del pantanal (Soares et al., 2013) y en la región amazónica brasileña (Majer \& Delabie, 1994), donde para ambos estudios la mayor riqueza se presentó en las áreas de tierra firme, es decir, que no sufren inundaciones.

La riqueza de especies en un determinado hábitat, se correlaciona de forma directa con el tamaño, estructura y composición vegetal (Ribas \& Schoereder, 2007), pero también con las características del micro hábitat (Queiroz et al., 2013). Aquí, el hecho que la riqueza registrada fuera mayor en los bosques secundarios que en los primarios, puede tener una relación con el incremento frecuente y esporádico que acontece de hormigas en áreas que fueron sometidas a algún nivel de perturbación (Kaspari, 2003). Del mismo modo, el proceso denominado retrogresión del bosque primario, que se caracteriza por una disminución en la producción primaria neta, genera directa o indirectamente un declino de la diversidad y riqueza en el ecosistema (Wardle, 2006). Resultados similares fueron reportados por Mathieu et al. (2005) en la región Oriental Amazónica donde, utilizando el mismo método TSBF, encontró que la riqueza de especies de hormigas en bosques secundarios jóvenes y antiguos, la riqueza de especies (66) era muy próxima de la riqueza registrada en los bosques primarios (76).

Las curvas de rarefacción en general no presentaron estabilización con tendencia asintótica, lo que sugiere un incremento en el esfuerzo de muestreo. El hecho que no se hubiese logrado la estabilización se puede estar presentando por la elevada riqueza de especies (Jiménez-Valverde \& Hortal, 2003; Leponce, Theunis, Delabie, \& Roisin 2004), y la ocurrencia de especies raras que albergan los suelos en la región tropical (Laurance \& Vasconcelos, 2009; Rousseau et al., 2014); por tal razón, el esfuerzo de muestreo para estimar la riqueza de especies debería ser mayor.

En el marco de la conservación y valoración biológica, el uso de taxones superiores como sustitutos para estimar la riqueza de especies invertebradas ya ha sido evaluada. Por ejemplo, en Araneae Cardoso et al. (2004), en Hymenoptera, Vieira et al. (2012), Coleoptera, Diptera y Acari (Báldi, 2003). En estos estudios, al igual que en este trabajo, se registró el género como el mejor sustituto para la estimación de la riqueza de especies.

Este trabajo ratifica los resultados obtenidos por Andersen (1995) en Australia, donde la relación entre la riqueza de especies de hormigas y riqueza de géneros es fuerte dentro de una misma región. De otro lado, diversos estudios verificaron que esta relación no solo es fuerte dentro de la región, sino también entre regiones con diversos tipos de hábitats, por ejemplo, Groc et al. (2010) en su trabajo realizado con datos sobre hormigas de Centro y Sur América, sugieren el género como el nivel que mayor información suministra. De hecho, en los Himalayas indianos con una variación altitudinal entre 1400 y $3700 \mathrm{msnm}$ Negi y Gadgil (2002), encontraron de manera similar, que el taxón que mejor explica la riqueza de especies en hormigas es el género. Souza et al. (2015) en la región amazónica central, evidenciaron que la localización y el tipo de vegetación no tuvieron un efecto significativo sobre la relación entre la riqueza de especies de hormigas y taxones sustitutos. Además, estimaron una reducción en costos totales del $40 \%$ cuando utilizaron el nivel de género en lugar de especies. Nuestro trabajo ha ampliado este patrón general, lo que demuestra que el género también puede ser un excelente sustituto de especies de hormigas en los bosques amazónicos orientales en diferente estado de sucesión vegetal.

De modo general, los trabajos anteriores sugieren la utilización del género como sustituto para la estimación rápida de la riqueza de especies, siempre y cuando las condiciones 
para la identificación de las especies no sean posibles o necesarias. En otros términos, si existe la necesidad de identificar especies "bioindicadoras" de perturbaciones mínimas en el ambiente (Nahmani, Lavelle, \& Rossi, 2006) o mapear las ocurrencias de especies raras (Heino \& Soininen, 2007), esta herramienta no sería la más adecuada. De hecho, mudanzas sutiles en la riqueza y composición de especies invertebrados del suelo pueden no ser detectadas en niveles superiores (Groc et al., 2010).

Entretanto, los resultados muestran al igual que en otras investigaciones realizadas con hormigas (Rosser \& Eggleton, 2012; Souza et al., 2015), y en otros grupos de organismos como, mamíferos (Grelle, 2002) y arañas (Cardoso et al., 2004), que la subfamilia no se comporta como un sustituto adecuado, por tanto, no debería ser utilizada. Esta respuesta podría tener relación con la escala taxonómica. De acuerdo con Vieira et al. (2012), taxones más elevados podrían no ser unidades naturales y por ende no necesariamente evolutivamente relacionados.

A pesar que la región oriental amazónica presenta una degradación acelerada de sus ecosistemas (Rousseau et al., 2014), no existe claridad ni dimensión sobre la contribución de los bosques secundarios como refugio para las especies de menor tamaño (Bihn, Verhaagh, Brändle, \& Brandl, 2008). De aquí que, tanto la conservación de los remanentes forestales como los bosques secundarios, presentan un potencial enorme para la conservación de las especies de hormigas y la sustentabilidad de la región (Mathieu et al., 2005; Rousseau et al., 2014). Las hormigas son ingenieras del ecosistema (Lavelle et al., 2006) y por esto merecen especial atención con el fin de garantizar las funciones ecológicas.

Aunque la planificación y ejecución de programas de monitoreo y conservación de la biodiversidad deben ser basadas principalmente a nivel de especie, el enfoque de taxones sustitutos es una herramienta que permite valorar la riqueza de hormigas en los sistemas agroforestales y diferentes estados de sucesión del bosque. Sin embargo, se sugiere solo bajo condiciones en las cuales sea imposible trabajar con el nivel de especie.

\section{AGRADECIMIENTOS}

A la Coordinación de Perfeccionamiento de Personal de Nivel Superior - CAPES por la beca concebida. A la Fundación de Amparo a la Investigación y Desenvolvimiento Científico y Tecnológico de Maranhão - FAPEMA por el apoyo financiero. A Fabricio Baccaro e Danielle Celentano por sus sugerencias al manuscrito. A todos los agricultores (as) y estudiantes que ayudaron durante el trabajo de campo y laboratorio. JHCD agradece su beca de investigación al CNPq.

\section{RESUMEN}

La deforestación es una de las principales causas de pérdida de biodiversidad en todo el mundo. Las hormigas son clave como ingenieras del ecosistema, por lo tanto, la pérdida de la diversidad de hormigas puede indicar la pérdida de funciones cruciales de los ecosistemas. El objetivo de este estudio fue evaluar la riqueza de hormigas del suelo y estimar si los niveles superiores (Subfamilia y Género) pueden ser usados como sustitutos para estimar la riqueza de especies en diferentes estados de sucesión del bosque (bosque primario, bosque secundario y sistema agroforestal) en la Amazonía Oriental. Fueron muestreadas 65 parcelas en el estado de Maranhão y Pará entre 2011 y 2014. El esquema de muestreo siguió el procedimiento de Biología de Suelos Tropicales y Fertilidad (TSBF). Primero caracterizamos los tipos de vegetación de acuerdo con su edad y luego se estimó la riqueza de especies. Para evaluar si los taxones superiores pueden utilizarse como sustitutos utilizamos funciones lineales y exponenciales y correlación de Pearson. En total, se identificaron 180 especies distribuidas en 60 géneros. Los resultados mostraron que la riqueza fue superior en el bosque secundario intermedio (88) y avanzado (76) y fue menor en el sistema agroforestal (38) y bosque ribereño primario (35). El género fue el mejor sustituto para estimar la riqueza de especies de hormigas a través de los diferentes tipos de sucesión vegetal, explicando entre el 72-97\% $(\mathrm{P}<0.001)$ de la variabilidad total de especies. Nuestros resultados confirman que el uso del nivel de género es un excelente sustituto para estimar la riqueza de especies de hormigas en la región y tanto 
los bosques en regeneración y sistemas agroforestales pueden contribuir en la conservación de la comunidad de hormigas en la Amazonía Oriental.

Palabras clave: taxones sustitutos, conservación, evaluación rápida de riqueza, Amazonía, taxonomía.

\section{REFERENCIAS}

Almeida, A. S. \& Vieira, I. C. (2010). Centro de endemismo Belém: status da vegetação remanescente e desafios para a conservação da biodiversidade e restauração ecológica. Revista de Estudos Universitários, 36, 95-111.

Alvares, C. A., Stape, J. L., Sentelhas, P. C., MoraesGonçalves, J. L., \& Sparovek, G. (2013). Köppen's climate classification map for Brazil. Meteorologische Zeitschrift, 22, 711-728.

Andersen, A. N. (1995). Measuring more of biodiversity: genus richness as a surrogate for species richness in Australian ant faunas. Biological Conservation, $73,39-43$.

Anderson, J. D., \& Ingram, J. S. I. (1993). Tropical soil biology and fertility: a handbook of methods (2 ed.). Wallingford: $\mathrm{CAB}$ International.

Antwiki.org. (2015). Distribution and Diversity. (Recuperado de http://www.antwiki.org/wiki/ Diversity_by_Country).

Báldi, A. (2003). Using higher taxa as surrogates of species richness: a study based on 3700 Coleoptera, Diptera, and Acari species in Central-Hungarian reserves. Basic and Applied Ecology, 4, 589-593.

Bihn, J. H., Verhaagh, M., Brändle, M., \& Brandl, R. (2008). Do secondary forests act as refuges for old growth forest animals? Recovery of ant diversity in the Atlantic forest of Brazil. Biological Conservation, $141,733-743$.

Cardoso, P., Silva, I., de Oliveira, N. G., \& Serrano, A. R. (2004). Higher taxa surrogates of spider (Araneae) diversity and their efficiency in conservation. Biological Conservation, 117, 453-459.

Chanatásig-Vaca, C. I., Huerta, E., Rojas, P., PonceMendoza, A., Mendoza, J., Morón, A., Van der wal, H., \& Dzib-Castillo, B. B. (2011). Efecto del uso de suelo en las hormigas (Formicidae: Hymenoptera) de Tikinmul, Campeche, México. Acta Zoológica Mexicana, 27, 441-461.

Coleman, B. D. (1981). On random placement and species-area relations. Mathematical Biosciences, 54, 191-215.

Colwell, R. K. (2013). Estimates: Statistical Estimation of Species Richness and Shared Species from Samples.
Version 9.1.0. User's Guild and application. Recuperado de http:// viceroy.eeb.uconn.edu/estimates

Colwell, R. K., Chao, A., Gotelli, N. J., Lin, S. Y., Mao, C. X., Chazdon, R. L., \& Longino, J. T. (2012). Models and estimators linking individual-based and samplebased rarefaction, extrapolation and comparison of assemblages. Journal of Plant Ecology, 5, 3-21.

Fearnside, P. M. (2006). Desmatamento na Amazônia: dinâmica, impactos e controle. Acta Amazonica, 36, 395-400.

Fearnside, P. M. (2010). Consequências do desmatamento da Amazônia. Scientific American Brasil Especial Biodiversidade, 3, 54-59.

Fernández, F. (2003). Introducción a las hormigas de la región Neotropical. Bogotá: Instituto de Investigación de Recursos Biológicos Alexander Von Humboldt.

Freitas, J. M. S., Delabie, J. H. C., \& Lacau, S. (2014). Composition and diversity of ant species into leaf litter of two fragments of a semi-deciduous seasonal forest in the Atlantic forest biome in Barra do Choça, Bahia, Brazil. Sociobiology, 61, 9-20.

Folgarait, P. J. (1998). Ant biodiversity and its relationship to ecosystem functioning: A review. Biodiversity and Conservation, 7, 1221-1244.

Fowler, H. G., Forti, L. C., Brandão C. R. F., Delabie J. H. C., \& Vasconcelos, H. L. (1991). Ecologia nutricional de formigas (pp. 131-209). En A. R. Pazzini \& J. R. P. Parra (Eds.), Ecologia nutricional de insetos e suas implicações no manejo de pragas. São Paulo, Manole.

Gaston, K. J., \& Williams, P. H. (1993). Mapping the world's species-the higher taxon approach. Biodiversity Letters, 1, 2-8.

Grelle, C. E. V. (2002). Is higher-taxon analysis an useful surrogate of species richness in studies of Neotropical mammal diversity? Biological Conservation, 108, 101-106.

Groc, S., Delabie, J. C. H., Longino, J. T., Orivel, J., Majer, J. D., Vasconcelos, H. L., \& Dejean, A. (2010). A new method based on taxonomic sufficiency to simplify studies on Neotropical ant assemblages. Biological Conservation, 143, 2832-2839.

Guzmán-Mendoza, R., Castaño-Meneses, G., \& NuñezPalenius, H. (2016). The diversity of ant communities (Hymenoptera: Formicidae) and their connections with other arthropods from three temperate forests of Central Mexico. Revista de Biología Tropical, 64, 571-585. (Doi: http://dx.doi.org/10.15517/rbt. v64i2.18979).

Heino, J., \& Soininen, J. (2007). Are higher taxa adequate surrogates for species-level assemblage patterns and 
species richness in stream organisms? Biological Conservation, 137, 78-89.

Hölldobler, B., \& Wilson, E. O. (1990). The Ants. Cambridge: Harvard University Press.

IBGE. (2011). Mapa tematicos vegetação. Instituto Brasileiro de Geografia e Estatística, Brasil. Recuperado de http://mapas.ibge.gov.br/tematicos/vegetacao.html

INPE. (2015). Instituto Nacional de Pesquisas Espaciais Monitoramento da Floresta Amazônica Brasileira por Satélite Projeto Prodes. Instituto Nacional de Pesquisas Espaciais, Brasil. Recuperado de http:// www.obt.inpe.br/prodes

Jiménez-Valverde, A., \& Hortal, J. (2003). Las curvas de acumulación de especies y la necesidad de evaluar la calidad de los inventarios biológicos. Revista Ibérica de Aracnología, 8, 151-161.

Kallimanis, A. S., Mazaris, A. D., Tsakanikas, D., Dimopoulos, P., Pantis, J. D., \& Sgardelis, S. P. (2012). Efficient biodiversity monitoring: Which taxonomic level to study? Ecological Indicators, 15, 100-104.

Kaspari, M. (2003). Introducción a la ecología de las hormigas. En F. Fernández (Ed.), Introducción a las Hormigas de la región Neotropical (pp. 97-112). Bogotá, Colombia: Instituto de Investigación de Recursos Biológicos Alexander von Humboldt.

Lattke, J. E. (2003). Biogeografía de las hormigas neotropicales. En F. Fernández (Ed.), Introducción a las Hormigas de la región Neotropical (pp. 65-88). Bogotá, Colombia: Instituto de Investigación de Recursos Biológicos Alexander von Humboldt.

Laurance, W. F., \& Vasconcelos, H. L. (2009). Consequências ecológicas da fragmentação florestal na amazônia. Oecologia Brasiliensis, 13, 434-451.

Lavelle, P., Decaëns, T., Aubert, M., Barot, S., Blouin, M., Bureau, F., Margerie, P., Mora, P., \& Rossi, J. P. (2006). Soil invertebrates and ecosystem services. European Journal of Soil Biology, 42, S3-S15.

Leal, I. R., Bieber, A. G. D., Tabarelli, M., \& Andersen, A. N. (2010). Biodiversity surrogacy: indicator taxa as predictors of total species richness in Brazilian Atlantic forest and Caatinga. Biodiversity and Conservation, 19, 3347-3360.

Leponce, M., Theunis, L., Delabie, J. H. C., \& Roisin, Y. (2004). Scale dependence of diversity measures in a leaf-litter ant assemblage. Ecography, 27, 253-267.

Majer, J. D., \& Delabie, J. H. C. (1994). Comparison of the ant communities of annually inundated and terra firme forests at Trombetas in the Brazilian Amazon. Insectes Sociaux, 41, 343-359.

Mathieu, J., Rossi, J. P., Mora, P., Lavelle, P., Martins, P. D. S., Rouland, C., \& Grimaldi, M. (2005). Recovery of soil macrofauna communities after forest clearance in Eastern Amazonia, Brazil. Conservation Biology, 19, 1598-1605.

Miranda, P. N., Oliveira, M. A., Baccaro, F. B., Morato, E. F., \& Delabie, J. H. C. (2012). Check list of grounddwelling ants (Hymenoptera: Formicidae) of the eastern Acre, Amazon, Brazil. CheckList, 8, 722-730.

Nahmani, J., Lavelle, P., \& Rossi, J. P. (2006). Does changing the taxonomical resolution alter the value of soil macroinvertebrates as bioindicators of metal pollution? Soil Biology and Biochemistry, 38, 385-396.

Neeson, T. M., Van Rijn, I., \& Mandelik, Y. (2013). How taxonomic diversity, community structure, and sample size determine the reliability of higher taxon surrogates. Ecological Applications, 23, 1216-1225.

Negi, H. R., \& Gadgil, M. (2002). Cross-taxon surrogacy of biodiversity in the Indian Garhwal Himalaya. Biological Conservation, 105, 143-155.

Palacio, E. E., \& Fernández, F. (2003). Claves para las subfamilias y géneros. En F. Fernández (Ed.), Introducción a las Hormigas de la región Neotropical (pp. 233-260). Bogotá, Colombia: Instituto de Investigación de Recursos Biológicos Alexander von Humboldt.

Pais, M. P., \& Varanda, E. M. (2010). Arthropod recolonization in the restoration of a semideciduous forest in southeastern Brazil. Neotropical Entomology, 39, 198-206.

Queiroz, A. C. M., Ribas, C. R., \& França, F. M. (2013). Microhabitat characteristics that regulate ant richness patterns: the importance of leaf litter for epigaeic ants. Sociobiology, 60, 367-373.

Quesada, C. A., Lloyd, J., Anderson, L. O., Fyllas, N. M., Schwarz, M., \& Czimczik, C. I. (2011). Soils of Amazonia with particular reference to the RAINFOR sites. Biogeosciences, 8, 1415-1440.

Ribas, C. R., \& Schoereder, J. H. (2007). Ant communities, environmental characteristics and their implications for conservation in the Brazilian Pantanal. Biodiversity and Conservation, 16, 1511-1520.

Ribas, C. R., Solar, R. R., Campos, R. B., Schmidt, F. A., Valentim, C. L., \& Schoereder, J. H. (2012). Can ants be used as indicators of environmental impacts caused by arsenic? Journal of Insect Conservation, $16,413-421$.

Rosser, N., \& Eggleton, P. (2012). Can higher taxa be used as a surrogate for species-level data in biodiversity surveys of litter/soil insects? Journal of Insect Conservation, 16, 87-92.

Rousseau, G. X., Rogério, P., Celentano, D., \& Carvalho, C. J. R. (2014). Macrofauna do solo em uma cronosequência de capoeiras, florestas e pastos no Centro 
de Endemismo Belém, Amazônia Oriental. Acta Amazonica, 44, 499-512.

StatSoft, Inc. (2002). STATISTICA 7.0 for Windows (Computer Program Manual). Tulsa, Oklahoma, USA: StatSoft.

Sanabria, C., Lavelle, P., \& Fonte, S. J. (2014). Ants as indicators of soil-based ecosystem services in agroecosystems of the Colombian Llanos. Applied Soil Ecology, 84, 24-30.

Santos, M. S., Louzada, J. N., Dias, N., Zanetti, R., Delabie, J. H. C., \& Nascimento, I. C. (2006). Riqueza de formigas (Hymenoptera, Formicidae) da serapilheira em fragmentos de floresta semidecídua da Mata Atlântica na região do Alto do Rio Grande, MG, Brasil. Iheringia, Série Zoologia, 96, 95-101.

Sebastia, H., \& Grelle, C. E. V. (2009). Taxon surrogates among Amazonian mammals: Can total species richness be predicted by single orders? Ecological Indicators, 9, 160-166.

Soares, S. D. A., Suarez, Y. R., Fernandes, W. D., Tenório, P. M. S., Delabie, J. H. C., \& Antonialli-Junior, W. F. (2013). Temporal variation in the composition of ant assemblages (Hymenoptera, Formicidae) on trees in the Pantanal floodplain, Mato Grosso do Sul, Brazil. Revista Brasileira de Entomologia, 57, 84-90.

Souza, J. L. P., Baccaro, F. B., Landeiro, V. L., Franklin, E., Magnusson, W. E., Pequeno, P. A. C. L., \& Fernandes, I. O. (2015). Taxonomic sufficiency and indicator taxa reduce sampling costs and increase monitoring effectiveness for ants. Diversity and Distributions, 22, 111-112.
Vasconcelos, H. L. (1999). Effects of forest disturbance on the structure of ground-foraging ant communities in central Amazonia. Biodiversity \& Conservation, $8,409-420$.

Vasconcelos, H. L., \& Laurance, W. F. (2005). Influence of habitat, litter type, and soil invertebrates on leaf-litter decomposition in a fragmented Amazonian landscape. Oecologia, 144, 456-462.

Vergara-Navarrro E. V., \& Serna, F. (2013). A checklist of the ants (Hymenoptera: Formicidae) of the department of Antioquia, Colombia, and new records for the country. Agronomía Colombiana, 31, 324-342.

Vieira, L. C., Oliveira, N. G., Brewster, C. C., \& Gayubo, S. F. (2012). Using higher taxa as surrogates of species-level data in three Portuguese protected areas: a case study on Spheciformes (Hymenoptera). Biodiversity and Conservation, 21, 3467-3486.

Wardle, D. A. (2006). The influence of biotic interactions on soil biodiversity. Ecology Letters, 9, 870-886.

Wilkie, K. R., Mertl, A. L., \& Traniello, J. F. (2010). Species diversity and distribution patterns of the ants of Amazonian Ecuador. PLoS One, 5, e13146.

Yepes, A. P., del Valle, J. I., Jaramillo, S. L., \& Orrego, S. A. (2010). Recuperación estructural en bosques sucesionales andinos de Porce (Antioquia, Colombia). Revista de Biología Tropical, 58, 427-445.

Zelarayán, M. L., Celentano, D., Oliveira, E. C., Triana, S. P., Sodré, D. N., Muchavisoy, K. H. M., \& Rousseau, G. X. (2015). Impact of degradation on carbon stock of riparian forests in the eastern Amazon, Brazil. Acta Amazonica, 45, 271-282. 


\section{APÉNDICE}

Municipios, estados de sucesión vegetal del bosque y sistemas agroforestales muestreados en la Amazonía Oriental, Brasil

\section{APPENDIX I}

Municipality, chronosequence of fallows, old-growth forests and agroforestry systems sampled in the Eastern Amazon, Brazil

\begin{tabular}{|c|c|c|c|c|c|}
\hline Municipio & Sucesión vegetal & Sigla & Edad & Coordenadas & $\mathrm{N}^{\circ}$ parcelas \\
\hline \multirow[t]{2}{*}{ Axixá } & Bosque secundario intermedio & BSI & 15 & $02^{\circ} 51^{\prime} 40^{\prime \prime} \mathrm{S}-44^{\circ} 08^{\prime} 00^{\prime \prime} \mathrm{W}$ & 1 \\
\hline & Bosque secundario avanzado & BSA & 60 & $02^{\circ} 52^{\prime} 21^{\prime \prime} \mathrm{S}-44^{\circ} 07^{\prime} 38^{\prime \prime} \mathrm{W}$ & 1 \\
\hline \multirow[t]{11}{*}{ Rosário } & Bosque secundario avanzado & BSA & 30 & $02^{\circ} 49^{\prime} 05^{\prime \prime} \mathrm{S}-44^{\circ} 08^{\prime} 20^{\prime \prime} \mathrm{W}$ & 3 \\
\hline & & & $>30$ & $02^{\circ} 51^{\prime} 04^{\prime \prime} \mathrm{S}-44^{\circ} 08^{\prime} 52^{\prime \prime} \mathrm{W}$ & \\
\hline & & & 30 & $02^{\circ} 51^{\prime} 11^{\prime \prime} \mathrm{S}-44^{\circ} 09^{\prime} 02^{\prime} \mathrm{W}$ & \\
\hline & Bosque secundario joven & BSJ & 5 & $02^{\circ} 51^{\prime} 02^{\prime \prime} \mathrm{S}-44^{\circ} 08^{\prime} 57^{\prime \prime} \mathrm{W}$ & 2 \\
\hline & & & 5 & $02^{\circ} 51^{\prime} 46^{\prime \prime} \mathrm{S}-44^{\circ} 08^{\prime} 32^{\prime \prime} \mathrm{W}$ & \\
\hline & Bosque secundario intermedio & BSI & 15 & $02^{\circ} 51^{\prime} 11^{\prime \prime} \mathrm{S}-44^{\circ} 09^{\prime} 30^{\prime \prime} \mathrm{W}$ & 3 \\
\hline & & & 15 & $02^{\circ} 51^{\prime} 17^{\prime \prime} \mathrm{S}-44^{\circ} 09^{\prime} 16^{\prime \prime} \mathrm{W}$ & \\
\hline & & & 15 & $02^{\circ} 51^{\prime} 34^{\prime \prime} \mathrm{S}-44^{\circ} 08^{\prime} 25^{\prime \prime} \mathrm{W}$ & \\
\hline & Bosque primario & $\mathrm{BP}$ & & $02^{\circ} 49^{\prime} 55^{\prime \prime} \mathrm{S}-44^{\circ} 07^{\prime} 56^{\prime \prime} \mathrm{W}$ & 1 \\
\hline & Sistema agroforestal & SAF & 9 & $02^{\circ} 51^{\prime} 14^{\prime \prime} \mathrm{S}-44^{\circ} 09^{\prime} 11^{\prime \prime} \mathrm{W}$ & 2 \\
\hline & & & $>30$ & $02^{\circ} 51^{\prime} 25^{\prime \prime} \mathrm{S}-44^{\circ} 08^{\prime} 03^{\prime \prime} \mathrm{W}$ & \\
\hline \multirow[t]{4}{*}{ São Luis } & Bosque secundario avanzado & BSA & 29 & $S-W$ & 1 \\
\hline & Bosque secundario joven & BSJ & 9 & $02^{\circ} 35^{\prime} 31^{\prime \prime} \mathrm{S}-44^{\circ} 12^{\prime} 36^{\prime \prime} \mathrm{W}$ & 3 \\
\hline & & & 7 & $02^{\circ} 35^{\prime} 25^{\prime \prime} \mathrm{S}-44^{\circ} 12^{\prime} 37^{\prime \prime} \mathrm{W}$ & \\
\hline & & & 7 & $02^{\circ} 35^{\prime} 26^{\prime \prime} \mathrm{S}-44^{\circ} 12^{\prime} 32^{\prime \prime} \mathrm{W}$ & \\
\hline \multirow[t]{2}{*}{ São José de Ribamar } & Bosque Restinga & $\mathrm{BR}$ & & $02^{\circ} 38^{\prime} 37^{\prime \prime} \mathrm{S}-44^{\circ} 08^{\prime} 17^{\prime \prime} \mathrm{W}$ & 2 \\
\hline & & & & $02^{\circ} 38^{\prime} 59^{\prime \prime} \mathrm{S}-44^{\circ} 84^{\prime} 10^{\prime \prime} \mathrm{W}$ & \\
\hline \multirow[t]{11}{*}{ Tomé- Açú } & Bosque secundario avanzado & BSA & 23 & $02^{\circ} 11^{\prime} 59^{\prime \prime} \mathrm{S}-48^{\circ} 08^{\prime} 45^{\prime \prime} \mathrm{W}$ & 2 \\
\hline & & & 20 & $02^{\circ} 24^{\prime} 48^{\prime \prime} \mathrm{S}-48^{\circ} 11^{\prime} 34^{\prime \prime} \mathrm{W}$ & \\
\hline & Bosque secundario joven & BSJ & 3 & $02^{\circ} 12^{\prime} 33^{\prime \prime} \mathrm{S}-48^{\circ} 09^{\prime} 28^{\prime \prime} \mathrm{W}$ & 2 \\
\hline & & & 4 & $02^{\circ} 12^{\prime} 34^{\prime \prime} \mathrm{S}-48^{\circ} 09^{\prime} 23^{\prime \prime} \mathrm{W}$ & \\
\hline & Bosque secundario intermedio & BSI & 12 & $02^{\circ} 11^{\prime} 55^{\prime \prime} \mathrm{S}-48^{\circ} 09^{\prime} 03^{\prime \prime} \mathrm{W}$ & 2 \\
\hline & & & 14 & $02^{\circ} 11^{\prime} 59^{\prime \prime} \mathrm{S}-48^{\circ} 08^{\prime} 58^{\prime \prime} \mathrm{W}$ & \\
\hline & Bosque primario & $\mathrm{BP}$ & & $02^{\circ} 24^{\prime} 40^{\prime \prime} \mathrm{S}-48^{\circ} 11^{\prime} 34^{\prime \prime} \mathrm{W}$ & 2 \\
\hline & & & & $02^{\circ} 39^{\prime} 24^{\prime \prime} \mathrm{S}-48^{\circ} 10^{\prime} 64^{\prime \prime} \mathrm{W}$ & \\
\hline & Sistema agroforestal & SAF & 18 & $02^{\circ} 11^{\prime} 55^{\prime \prime} \mathrm{S}-48^{\circ} 09^{\prime} 17^{\prime \prime} \mathrm{W}$ & 3 \\
\hline & & & 34 & $02^{\circ} 32^{\prime} 38^{\prime \prime} \mathrm{S}-48^{\circ} 16^{\prime} 26^{\prime \prime} \mathrm{W}$ & \\
\hline & & & 36 & $02^{\circ} 31^{\prime} 60^{\prime \prime} \mathrm{S}-48^{\circ} 10^{\prime} 63^{\prime \prime} \mathrm{W}$ & \\
\hline \multirow[t]{12}{*}{ Alcântara } & Bosque secundario ribereño joven & BSRJ & & $02^{\circ} 21^{\prime} 42^{\prime \prime} \mathrm{S}-44^{\circ} 26^{\prime} 31^{\prime \prime} \mathrm{W}$ & 6 \\
\hline & & & & $02^{\circ} 21^{\prime} 35^{\prime \prime} \mathrm{S}-44^{\circ} 27^{\prime} 26^{\prime \prime} \mathrm{W}$ & \\
\hline & & & & $02^{\circ} 21^{\prime} 02^{\prime \prime} \mathrm{S}-44^{\circ} 27^{\prime} 56^{\prime \prime} \mathrm{W}$ & \\
\hline & & & & $02^{\circ} 20^{\prime} 52^{\prime \prime} \mathrm{S}-44^{\circ} 28^{\prime} 39^{\prime \prime} \mathrm{W}$ & \\
\hline & & & & $02^{\circ} 20^{\prime} 55^{\prime \prime} \mathrm{S}-44^{\circ} 28^{\prime} 56^{\prime \prime} \mathrm{W}$ & \\
\hline & & & & $02^{\circ} 20^{\prime} 38^{\prime \prime} \mathrm{S}-44^{\circ} 29^{\prime} 10^{\prime \prime} \mathrm{W}$ & \\
\hline & Bosque secundario ribereño avanzado & BSRA & & $02^{\circ} 21^{\prime} 05^{\prime \prime} \mathrm{S}-44^{\circ} 27^{\prime} 51^{\prime \prime} \mathrm{W}$ & 6 \\
\hline & & & & $02^{\circ} 21^{\prime} 43^{\prime \prime} \mathrm{S}-44^{\circ} 26^{\prime} 36^{\prime \prime} \mathrm{W}$ & \\
\hline & & & & $02^{\circ} 21^{\prime} 37^{\prime \prime} \mathrm{S}-44^{\circ} 27^{\prime} 36^{\prime \prime} \mathrm{W}$ & \\
\hline & & & & $02^{\circ} 20^{\prime} 47^{\prime \prime} \mathrm{S}-44^{\circ} 28^{\prime} 29^{\prime \prime} \mathrm{W}$ & \\
\hline & & & & $02^{\circ} 20^{\prime} 53^{\prime \prime} \mathrm{S}-44^{\circ} 28^{\prime} 54^{\prime \prime} \mathrm{W}$ & \\
\hline & & & & $02^{\circ} 20^{\prime} 52^{\prime \prime} \mathrm{S}-44^{\circ} 29^{\prime} 01^{\prime \prime} \mathrm{W}$ & \\
\hline
\end{tabular}


APÉNDICE 1 (Continuación) / APPENDIX 1 (Continued)

\begin{tabular}{|c|c|c|c|c|c|}
\hline Municipio & Sucesión vegetal & Sigla & Edad & Coordenadas & $\mathrm{N}^{\circ}$ parcelas \\
\hline & Área abierta uso agrícola & AA & 1 & $02^{\circ} 21^{\prime} 39^{\prime \prime} \mathrm{S}-44^{\circ} 26^{\prime} 30^{\prime \prime} \mathrm{W}$ & 6 \\
\hline & & & 1 & $02^{\circ} 21^{\prime} 37^{\prime \prime} \mathrm{S}-44^{\circ} 27^{\prime} 16^{\prime \prime} \mathrm{W}$ & \\
\hline & & & 1 & $02^{\circ} 21^{\prime} 32^{\prime \prime} \mathrm{S}-44^{\circ} 27^{\prime} 43^{\prime \prime} \mathrm{W}$ & \\
\hline & & & 1 & $02^{\circ} 20^{\prime} 53^{\prime \prime} \mathrm{S}-44^{\circ} 28^{\prime} 50^{\prime \prime} \mathrm{W}$ & \\
\hline & & & 1 & $02^{\circ} 20^{\prime} 56^{\prime \prime} \mathrm{S}-44^{\circ} 29^{\prime} 01^{\prime \prime} \mathrm{W}$ & \\
\hline & & & 1 & $02^{\circ} 20^{\prime} 54^{\prime \prime} \mathrm{S}-44^{\circ} 28^{\prime} 46^{\prime \prime} \mathrm{W}$ & \\
\hline & Bosque ribereño primario & $\mathrm{BRP}$ & & $02^{\circ} 21^{\prime} 37^{\prime \prime} \mathrm{S}-44^{\circ} 26^{\prime} 54^{\prime \prime} \mathrm{W}$ & 6 \\
\hline & & & & $02^{\circ} 21^{\prime} 39^{\prime \prime} \mathrm{S}-44^{\circ} 27^{\prime} 14^{\prime \prime} \mathrm{W}$ & \\
\hline & & & & $02^{\circ} 20^{\prime} 49^{\prime \prime} \mathrm{S}-44^{\circ} 28^{\prime} 31^{\prime \prime} \mathrm{W}$ & \\
\hline & & & & $02^{\circ} 20^{\prime} 52^{\prime \prime} \mathrm{S}-44^{\circ} 28^{\prime} 50^{\prime \prime} \mathrm{W}$ & \\
\hline & & & & $02^{\circ} 20^{\prime} 36^{\prime \prime} \mathrm{S}-44^{\circ} 29^{\prime} 08^{\prime \prime} \mathrm{W}$ & \\
\hline & & & & $02^{\circ} 20^{\prime} 55^{\prime \prime} \mathrm{S}-44^{\circ} 27^{\prime} 56^{\prime \prime} \mathrm{W}$ & \\
\hline \multirow[t]{11}{*}{ Gurupi } & Bosque Secundario avanzado & BSA & $>28$ & $04^{\circ} 04^{\prime} 08^{\prime \prime} \mathrm{S}-46^{\circ} 53^{\prime} 22^{\prime \prime} \mathrm{W}$ & 2 \\
\hline & & & $>28$ & $04^{\circ} 04^{\prime} 09^{\prime \prime} \mathrm{S}-46^{\circ} 53^{\prime} 20^{\prime \prime} \mathrm{W}$ & \\
\hline & Bosque secundario intermedio & BSI & $>10$ & $04^{\circ} 02^{\prime} 10^{\prime \prime} \mathrm{S}-46^{\circ} 52^{\prime} 50^{\prime \prime} \mathrm{W}$ & 3 \\
\hline & & & $>10$ & $04^{\circ} 02^{\prime} 05^{\prime \prime} \mathrm{S}-46^{\circ} 52^{\prime} 51^{\prime \prime} \mathrm{W}$ & \\
\hline & & & 13 & $04^{\circ} 02^{\prime} 34^{\prime \prime} \mathrm{S}-46^{\circ} 54^{\prime} 21^{\prime \prime} \mathrm{W}$ & \\
\hline & Bosque primario & BP & & $03^{\circ} 40^{\prime} 50^{\prime \prime} \mathrm{S}-46^{\circ} 46^{\prime} 12^{\prime \prime} \mathrm{W}$ & 6 \\
\hline & & & & $03^{\circ} 40^{\prime} 56^{\prime \prime} \mathrm{S}-46^{\circ} 46^{\prime} 08^{\prime \prime} \mathrm{W}$ & \\
\hline & & & & $03^{\circ} 40^{\prime} 59^{\prime \prime} \mathrm{S}-46^{\circ} 46^{\prime} 01^{\prime \prime} \mathrm{W}$ & \\
\hline & & & & $03^{\circ} 41^{\prime} 11^{\prime \prime} \mathrm{S}-46^{\circ} 45^{\prime} 34^{\prime \prime} \mathrm{W}$ & \\
\hline & & & & $03^{\circ} 41^{\prime} 14^{\prime \prime} \mathrm{S}-46^{\circ} 45^{\prime} 34^{\prime \prime} \mathrm{W}$ & \\
\hline & & & & $03^{\circ} 41^{\prime} 16^{\prime \prime} \mathrm{S}-46^{\circ} 45^{\prime} 34^{\prime \prime} \mathrm{W}$ & \\
\hline
\end{tabular}


